\title{
Experimental Study on Acoustic Emission Characteristics of Phyllite Specimens under Uniaxial Compression
}

\author{
Z.Kui $^{1, *}$, Z.Zhicheng ${ }^{1}$, Z.Peng $^{2}$ and C.Sanjian ${ }^{1}$ \\ ${ }^{1}$ School of Resources \& Environment Engineering, Jiangxi University of Science and Technology, Ganzhou 341000, China \\ ${ }^{2}$ School of Civil and Environment Engineering, Beijing University of Science and Technology, Beijing 100083, China
}

Received 2 March 2015; Accepted 11 October 2015

\begin{abstract}
In order to obtain acoustic emission (AE) characteristics of phyllite specimens, AE tests of phyllite specimens under uniaxial compression were carried out. Results indicate that there are three distinct failure modes. The main mode is an extension mode. The others are a shear mode and a coupling of the shear and extension modes. A relative quiescence period of AE may appear before the peak stress for any failure mode. The ratio of the cumulative hits and the cumulative energy (named the $\mathrm{r}$ value and the Ib value respectively) are used for AE test results analysis. The plots of the Ib value show that the value variation is complex and usually decreases before the peak stress. Before the peak stress the $r$ value decreases continuously and then keeps a relatively slow variation. After the peak stress the $r$ value increases dramatically. The $r$ value appears to be in a U-shape distribution. The Ib value usually decreases while the $r$ value keeps a relatively low variation during the pre-peak stress stage, and the $r$ value increases sharply after the peak stress. This can be used as a basis for determining the pre- or post-peak stress state.
\end{abstract}

Keywords: Phyllite, Peak Stress, Residual Stress, Acoustic Emission, Failure Mode

\section{Introduction}

As acoustic emission (AE) can reflect the internal damage evolution in rock, concrete and other brittle materials, AE technology has been widely used to evaluate rock engineering stability [1],[2],[3]. A great deal of rock AE experiments have been carried out in the laboratory to investigate rock $\mathrm{AE}$ features in order to predict rock failure. These rock AE experiments have been studied for decades and mainly involve investigation of rock $\mathrm{AE}$ parameter variation regularity from loading to failure under different loading modes, and relations between rock AE parameters and micro-crack evolution laws and the damage process. For example, during $\mathrm{AE}$ experiments of granite and diabase samples under uniaxial compression the fractal features of the event rate and strength of AE were studied [4], [5]. The b value and fractal features of $\mathrm{AE}$ in the whole loading process of granite, marble and sandstone samples were analyzed in detail under uniaxial compression [6]. Moreover, Lu et al [7] proposed the $b$ value variation regularities during rock creep. Rock AE characteristics with micro-crack evolution have been investigated by many researchers. Lockner [8] discussed a correlation between the $\mathrm{AE}$ rate and inelastic strain by simply counting the number of $\mathrm{AE}$ events prior to sample failure. Stephen et al [9] pointed out that the AE event location has provided better information on the timing and position of failure based on numerous experiments. Relations between AE energy and frequency distribution with rock inner construction also investigated under uniaxial

* E-mail address: 296931654@qq.com ISSN: 1791-2377 (C) 2015 Kavala Institute of Technology. All rights reserved. conditions [10]. Experiments on the AE characteristics of the complete granite failure process were carried out under uniaxial multilevel loadings. Relations between $\mathrm{AE}$ characteristics and stress levels have also been obtained [11], The strength variation and $\mathrm{AE}$ characteristics of skarn under uniaxial cyclic loading and unloading have been studied. The results indicate that the uniaxial compression curve of skarn can be generally divided into four stages; each with different acoustic emission characteristics [12], The other researchers and the author have investigated the $\mathrm{AE}$ characteristics of rock and backfill body under cyclic loading [13], [14]. The comprehensive identification method of the rock AE Kaiser Effect has been proposed based on in situ and laboratory tests by the author [15].

Based on a great number of findings including those listed above, it has been shown that most researchers have focused on the relation between stress-strain and $\mathrm{AE}$ characteristics before the peak stress. Almost all efforts were made to seek the AE characteristics near to the peak stress for rock failure prediction. By comparison, there has been very little research involving AE pre- and post-peak stress, especially during residual stress periods. In this paper, I will focus primarily on understanding the complete rock failure behavior, including the post-peak region, through the use of $\mathrm{AE}$ and related techniques.

\section{Description of the problem}

It is common knowledge that rock pillars are the main support structure used to ensure the strength and stability of underground space in mining and other rock engineering works. These rock pillars, often bearing a strong load even after the peak stress, will still work as a support structure 
depending on their residual strength together with other support structures. In this case, a comparison study of the $\mathrm{AE}$ characteristics of pre- and post-peak stress can provide the basis to determine pillar stress state.

Phyllite pillars in underground mining often fail, and even break to some extent due to their schistosity plane, although they continue to play a supporting role. With this in mind, the pillar state in pre- or post-peak stress should be determined by AE monitoring. This paper studies the phyllite samples' stress state in pre- or post-peak stress by AE information analysis. Uniaxial compression experiments have been performed on 48 phyllite core samples taken from core boreholes drilled parallel to the direction of the schistosity plane at a lead-zinc underground mine in Jiangxi province, China. In all experiments, AE was monitored using an independent system that did not interfere with the uniaxial compression experiment.

\section{State of the art}

\section{1 b value}

Currently, the analysis method of AE signals mainly includes waveform analysis and parameter analysis. Waveform analysis aims to study the frequency and main energy distribution characteristics of $\mathrm{AE}$ signals under different stress states. For example, through experiments, Ji et al. (2012) proposed a prediction method for rock failure according to AE frequency characteristics under different stress states. Parameter analysis investigates the relation between time and AE parameters such as ringdown count, energy, amplitude, and so on. Researchers attempt to find the variation regularity of $\mathrm{AE}$ parameters during the prepeak stress period and use this as a guide for rock failure.

In parameter analysis, the relation between magnitude and frequency is commonly used, where the AE features are studied by the $\mathrm{b}$ value. In seismology, earthquakes of larger magnitudes occur less frequently than those of smaller magnitudes. Gutenberg and Richter noted that this relationship appears to obey a power law and obtained an empirical formula given by

$$
\log N=a+b M
$$

In the equation, $M$ is the earthquake magnitude, $N$ is the number of events with a magnitude greater than or equal to , is a measure of the regional level of seismicity, and is the so-called value. In terms of $\mathrm{AE}$ technique, is the $\mathrm{AE}$ amplitude dividing by a factor of 20, is the number of AE hits with amplitude greater than the threshold, and is an empirical constant. Different types of cracks generate different kinds of $\mathrm{AE}$ signals with varying frequency ranges and amplitudes. The $\mathrm{b}$ value represents the ratio of weak to strong events. The micro-cracks generate a large number of weak acoustic emissions, which leads to a relatively high $b$ value, while macro-cracking leads to relatively low $b$ values since they generate a large number of strong AE signals. Hence, a sharply decreasing b value can often be used as an indicator of near-peak stress [17],[18],[19].

\subsection{Experiment method}

The $b$ value was originally defined for the area of seismology. In the case of AE application, however, there are some problems to be solved. In order to apply the $b$ value to the $\mathrm{AE}$ technique, an improved $\mathrm{b}$ value (Ib value) is proposed [20]. To improve the calculation of the $b$ value, the number of $\mathrm{AE}$ data is formulated by

$\int_{0}^{\infty} n(\alpha) d(\alpha)=\beta$

In the equation, $n(\alpha)$ is the number of $\mathrm{AE}$ at $d(\alpha)$, and $\beta$ is the number of AE data.

Let $\mu$ be the mean of the amplitude distribution and $\sigma$ the standard deviation. The upper amplitude $w_{2}$ and lower amplitutde $w_{1}$ can then be formulated as $\mu+\alpha_{1} \sigma$ and $\mu-\alpha_{2} \sigma$ respectively. The following equations can be deduced:

$N\left(w_{1}\right)=N\left(\mu-\alpha_{2} \sigma\right)=\int_{\mu-\alpha_{2} \sigma}^{\infty} n(\alpha) d(\alpha)$

and

$N\left(w_{2}\right)=N\left(\mu+\alpha_{1} \sigma\right)=\int_{\mu+\alpha_{1} \sigma}^{\infty} n(\alpha) d(\alpha)$

In these equations, $N\left(w_{1}\right)$ is the accumulated number of $\mathrm{AE}$ events where amplitude is more than $\mu-\alpha_{2} \sigma$, and $N\left(w_{2}\right)$ is the accumulated number of $\mathrm{AE}$ events where amplitude is more than $\mu+\alpha_{l} \sigma$. Thus, the mathematical form of the $I b$ value is given as follows:

$I b=\frac{\lg N\left(w_{1}\right)-\lg N\left(w_{2}\right)}{\left(\alpha_{1}+\alpha_{2}\right) \sigma}$

In the equation, $\alpha_{1}$ and $\alpha_{2}$ are empirical constants; usually $\alpha_{1}$ is 1 and $\alpha_{2}$ is 0 .

An increasing trend for the $I b$ value exhibits the predominance of micro-fracture occurrence, and a decreasing trend for the $I b$ value demonstrates the predominance of macro-fracture occurrence. The $I b$ value could provide useful information concerning the state of the fracture and may be used to identify the failure process of a material during, inter alia, rock slope stability evaluations [20],[21],[22].

\section{$3.3 r$ value}

Based on cumulative hits and energy, Stephen et al. (2006) proposed a new ratio value (defined as the $r$ value in this paper) instead of the $b$ value.

$r=\frac{\sum N}{\sum E}$

In the equation, $\sum \mathrm{N}$ is cumulative hits and $\sum \mathrm{E}$ is the cumulative energy.

This ratio allows a combined interpretation of the two first-order AE parameters (hits and energy) without the need for line-fitting or windowing. The hypotheses for interpretation of the $r$ values are that an increase indicates the occurrence of numerous small, low-energy events, which could be associated with fracture propagation or shear fracturing, and a decrease indicates a small number of high energy events, which may occur at fracture initiation and particularly for opening mode ruptures [9]. The $r$ value together with the $I b$ value are used in this work to study the rock state in the pre- or post-peak stress period.

\section{Methodology}




\subsection{Experimental details}

All samples were of phyllite rock cored from the coring platform in the Yin Shan lead-zinc mine of Jiangxi province, China. They were mainly composed of sericite and silica. The cores from boreholes drilled parallel to the direction of the schistosity plane were chosen by their length being above $15 \mathrm{~cm}$ for convenient treatment, as shown in Figure 1. The cores were then cut to cylindrical dimensions with a diameter of $5 \mathrm{~cm}$ and a length of slightly more than $10 \mathrm{~cm}$ by a DQ-4A rock-cutting machine. Both ends of the rock samples were subsequently polished by an M-200-type double-end buffing machine. Finally, 48 cylinder specimens with dimensions of $50 \times 100 \mathrm{~mm}$ were formed which meet the experiment's requirements, as shown in Figure 2.

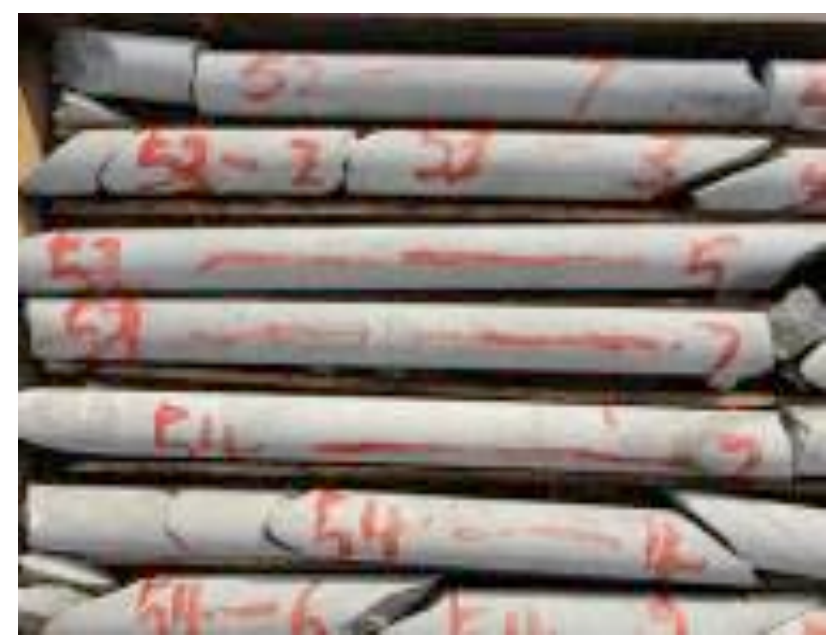

Fig.1. Part of the borehole cores. Self-Elaboration.

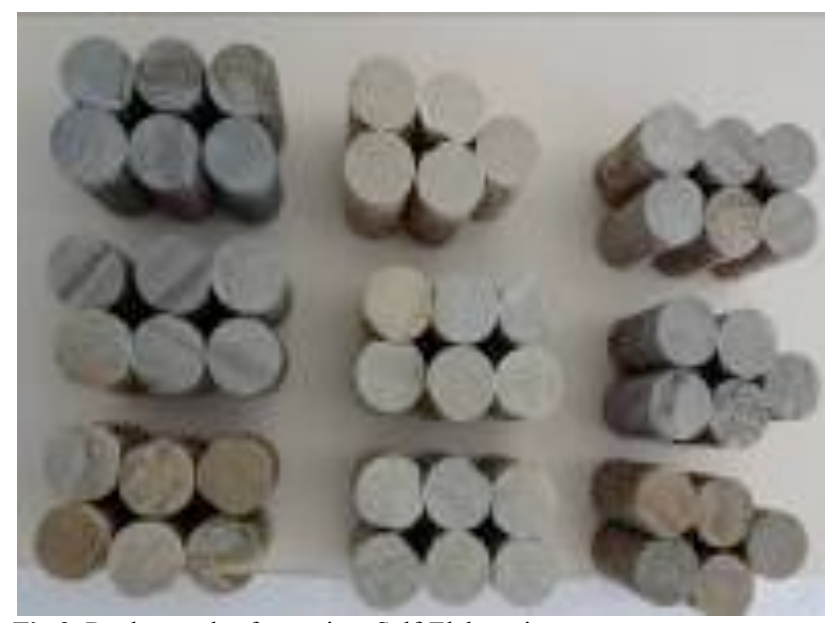

Fig.2. Rock samples for testing. Self-Elaboration

The experimental work uses the AE experiment system and the RMT-150C rock mechanics servo experiment system. The compression experiments were conducted with increasing axial displacement at a constant rate. A displacement rate of $5 \times 10-4 \mathrm{~mm} / \mathrm{s}$ was applied.

Acoustic measurements were performed using special hardware and software. The signals were recorded in a two channel monitoring board PCI-2, PAC. The sampling frequency was set to $1 \mathrm{Msps}$. The threshold was set to $45 \mathrm{db}$ to avoid noise, which is normal for the rock in question, and acquired signals were pre-amplified by another $45 \mathrm{db}$. Concerning AE monitoring, broadband $\mathrm{AE}$ sensors with maximum sensitivity at $400 \mathrm{kHz}$ were attached to the side of the specimen. Bearing grease was used to promote acoustic coupling, while the sensors were secured by special fixtures. In order to obtain all kinds of parameter variation with time, $\mathrm{AE}$ and the compression system should be working simultaneously. An image of the test system for AE under uniaxial compression is shown as Figure 3.

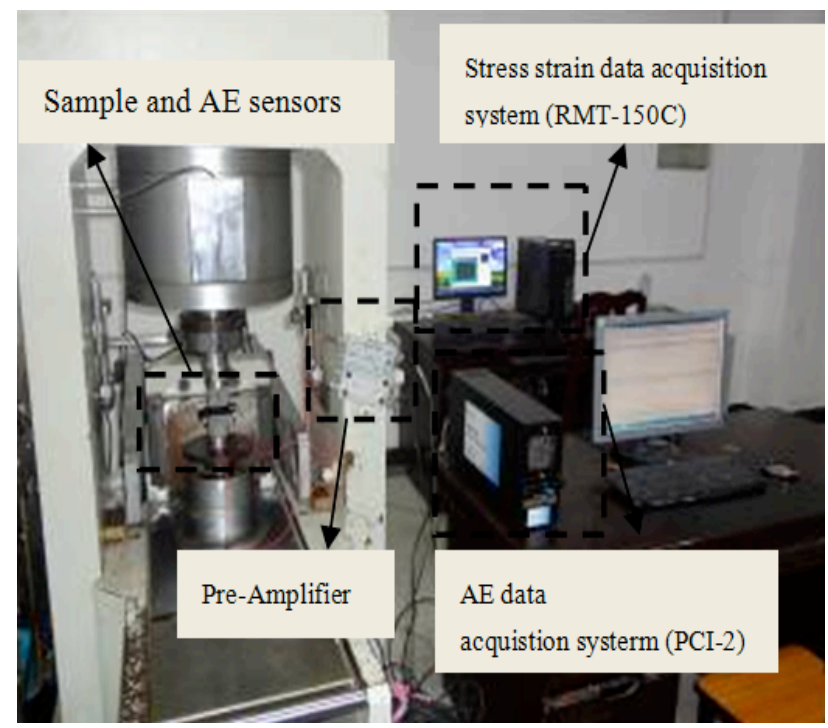

Fig.3. Image of test device for loading and AE. Self-Elaboration

\subsection{Mode of failure}

The macroscopic fractures of phyllite under uniaxial compression mainly occurred as samples fail along a plane which is almost parallel to the direction of compression, which is very similar to the experimental results of Zheng et al [23].

Three distinct modes of failure of the cylindrical samples were observed and identified: extension, shear and a coupling of the shear and extension modes. The extension mode denotes more fractures along a plane which is almost parallel to the direction of compression and is presented in Figure 4(a). For phyllite rock samples, an extension failure mode occurs predominantly due to the schistosity planes being nearly parallel to the direction of compression. Multiple extension failure of this kind occurs when planes are nearly parallel with each other. This differs from the multiple extension mode denoted by Szwedzicki [24], where failure planes may or may not be parallel. The second failure mode is typical shear mode, which always has one or more shear fracture planes, as shown in Figure 4(b). Failure samples show that shear planes are nearly parallel with each other. Clearly this kind of parallel plane can be seen as a determination of the phyllite rock structure. The third failure mode is called the coupling of the shear and extension modes, which has complicated extension-shear fracture planes, as shown in Figure 4(c). 

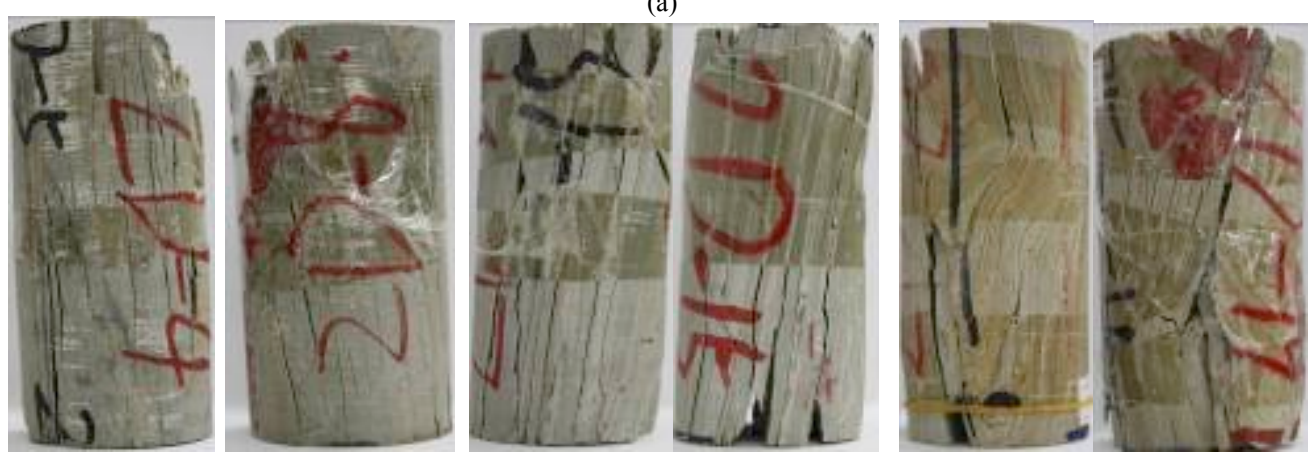

(b)
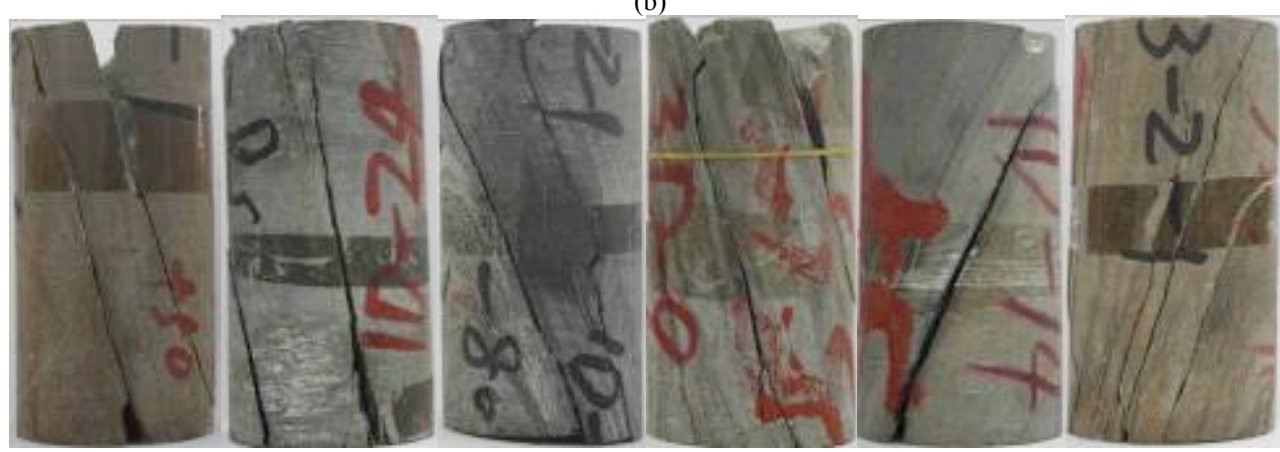

(c)
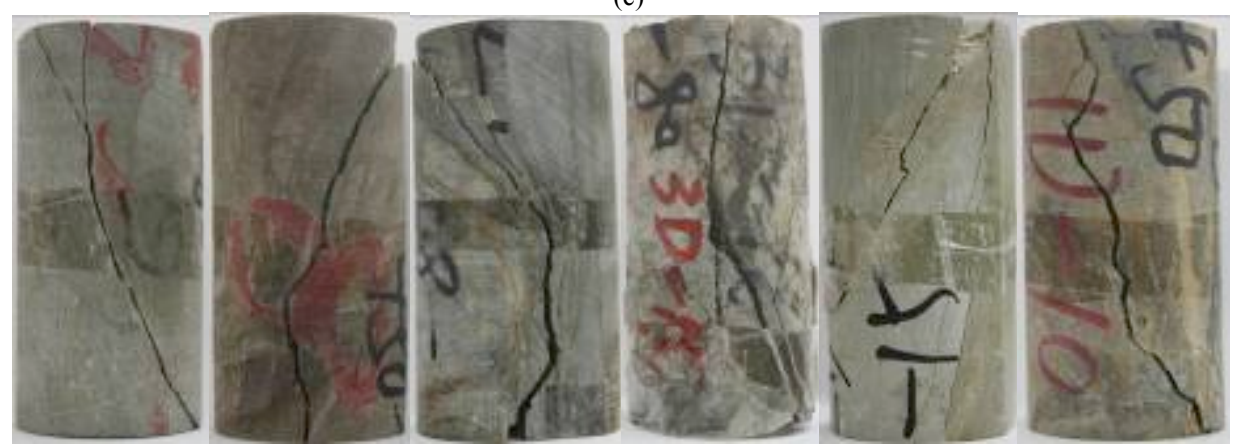

Fig.4. Typical failure modes. (a) extension mode; (b) shear mode; (c) coupling of the shear and extension modes. Self-Elaboration.

\subsection{AE features}

The experimental results show that phyllite rock samples have three failure modes although their AE features display no significant difference. The typical relations of stress and ringdown count rate changing with time are shown in figure 5 .

(a)

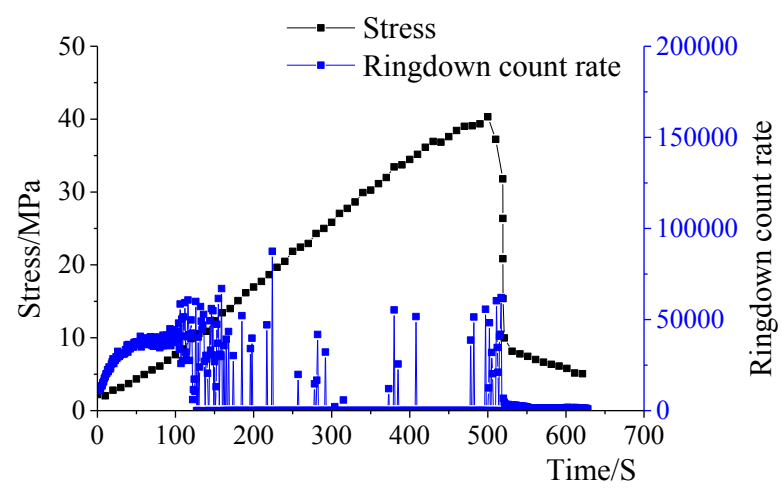

(b)

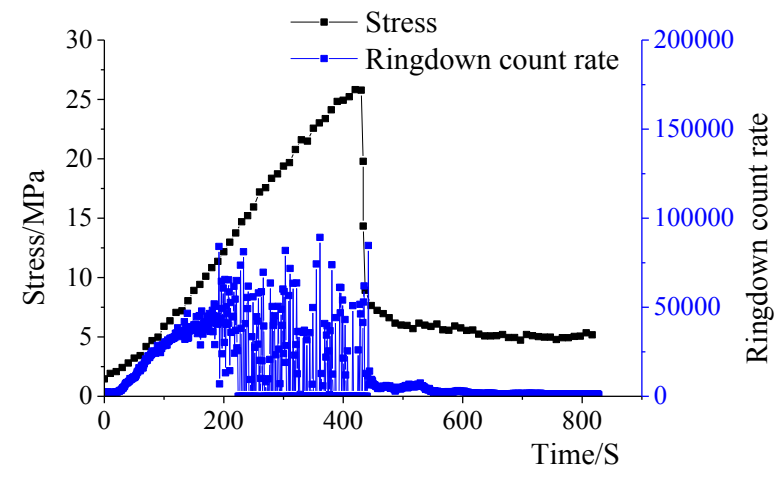

Fig. 5. Typical curves of stress and ringdown count rate changing with time. a) with a relative quiescence period; (b) without a relative quiescence period. Self-Elaboration.

We can see that AE occurs frequently when the stress is in a lower level period. Additionally, AE events reduce gradually with stress increase before peak stress. Some of the samples exhibit a relative quiescence period of $\mathrm{AE}$ before the peak stress. The phyllite rock sample is somewhat different from the AE features of other rock samples under 
uniaxial compression reported by the respective findings of other documents. The ringdown count rate does not increase sharply close to the peak stress, and especially after the peak stress (that is to say, during the residual stress phase) many AE events still emerge. Thus, comparing the AE characteristics of pre-peak and post-peak stress is an advantageous way of identifying the peak stress.

\section{Results}

\subsection{Failure Mode and its corresponding AE} Characteristics

After the experiment the recorded stress and AE data were processed. Using Eq.5 and Eq.6, the Ib value, $\mathrm{r}$ value and corresponding stress value changing with time were obtained, and the 18 typical results can be seen as Figures 6-8. In order to study the problem deeper, the $\mathrm{AE}$ characteristics will be discussed for the three different failure modes respectively.

\section{(a)}

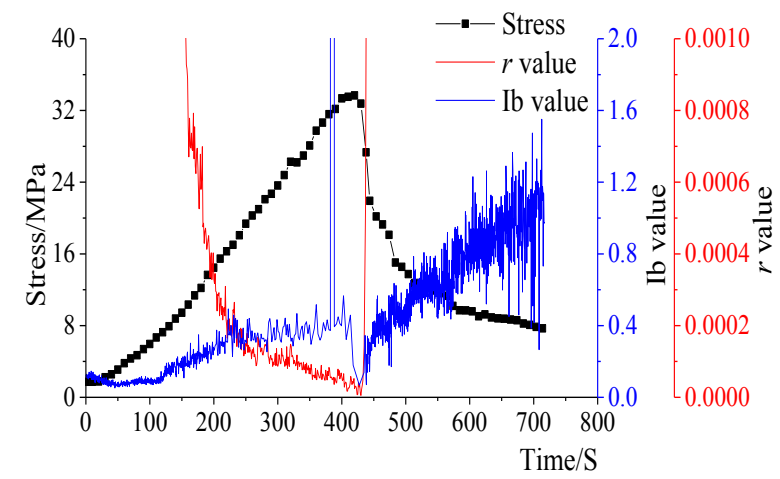

(b)

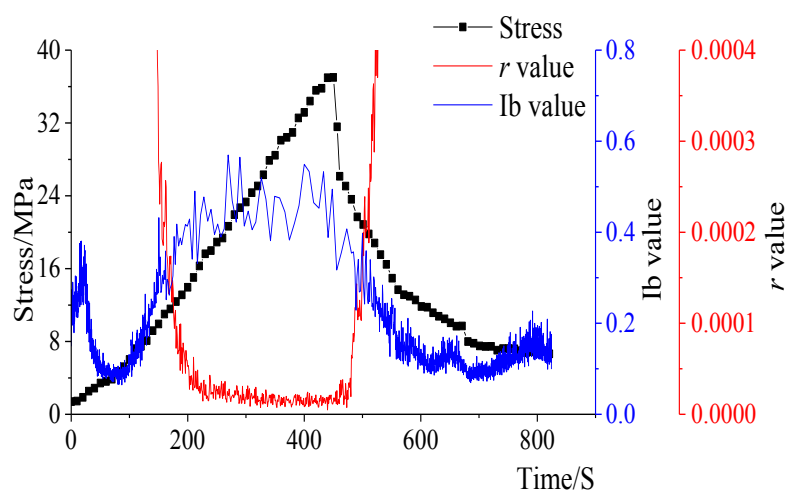

(c)

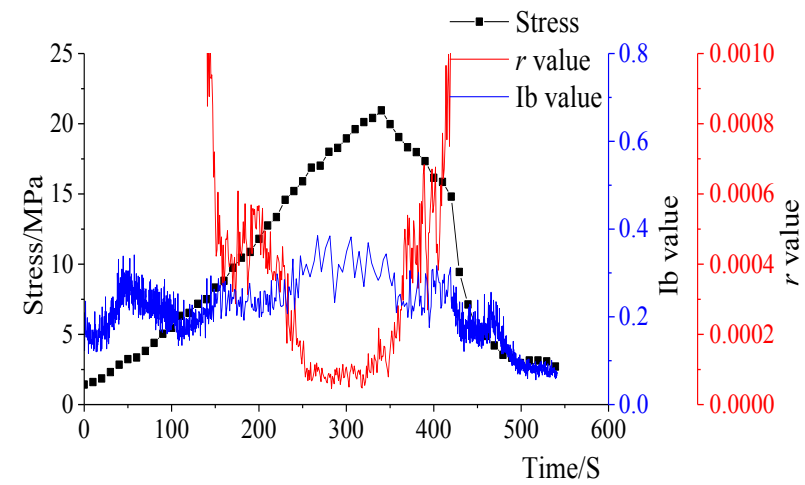

(d)

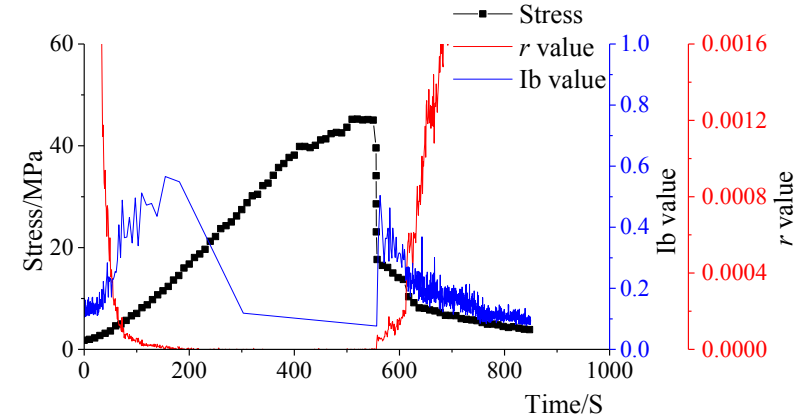

(e)

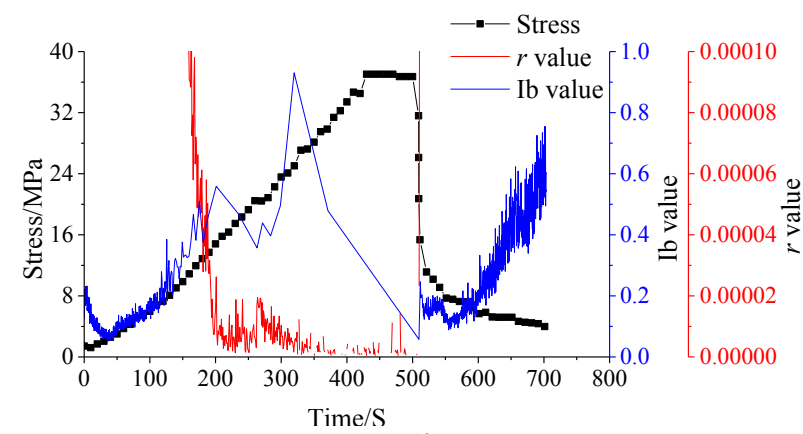

(f)

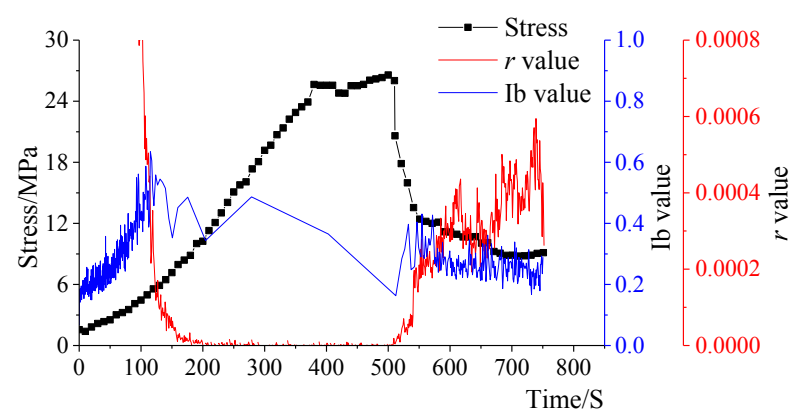

Fig. 6. Relationship between stress and AE parameters of samples in the extension mode. Self-Elaboration

(a)

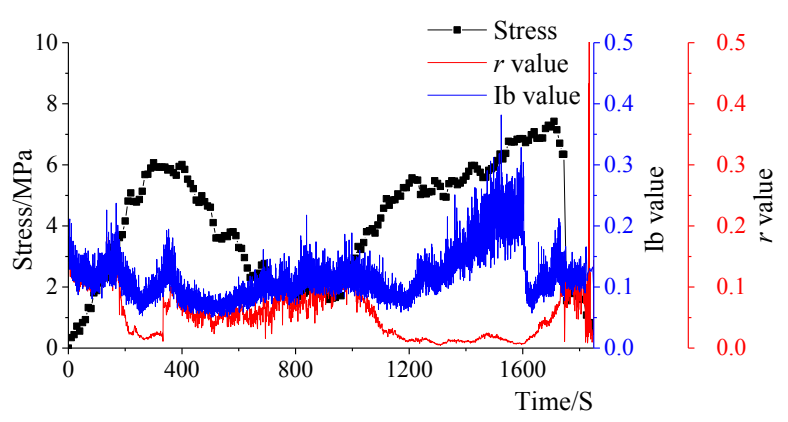

(b)

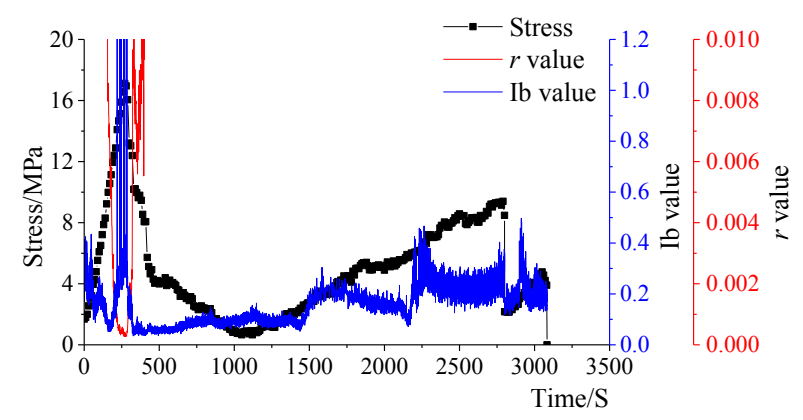

(c) 


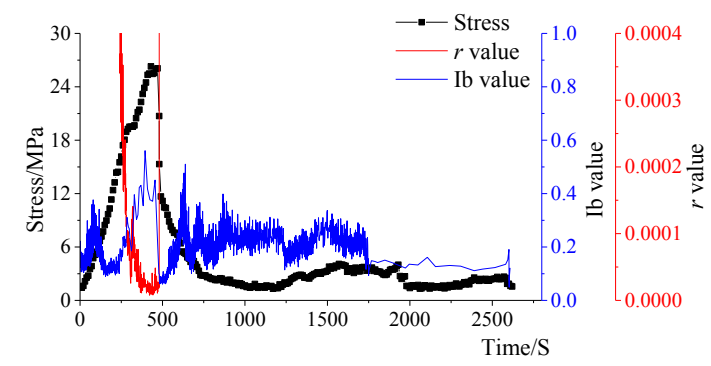

(d)

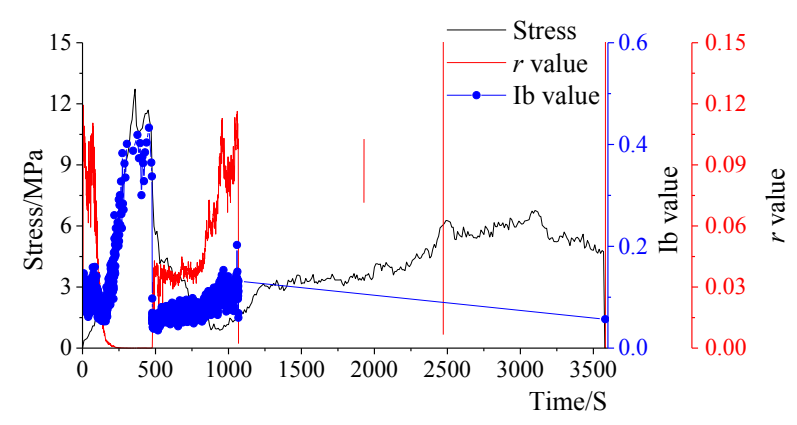

(e)

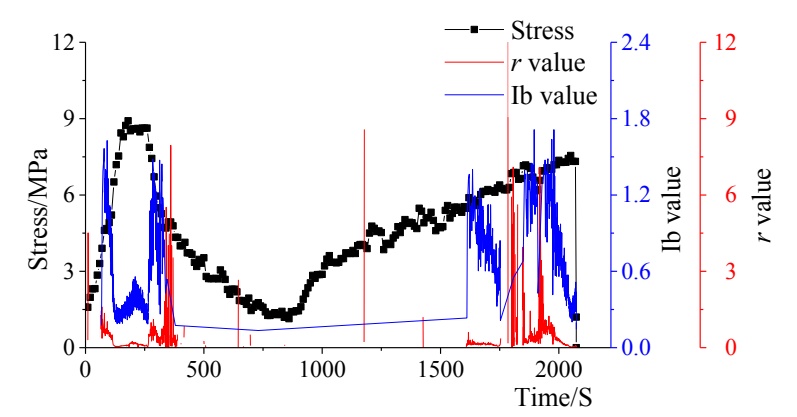

(f)

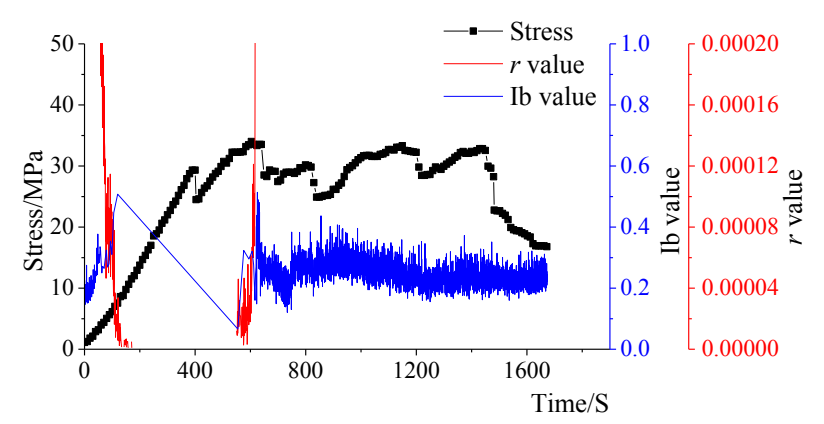

Fig. 7. Relationship between stress and AE parameters of samples in the shear mode. Self-Elaboration.

(a)

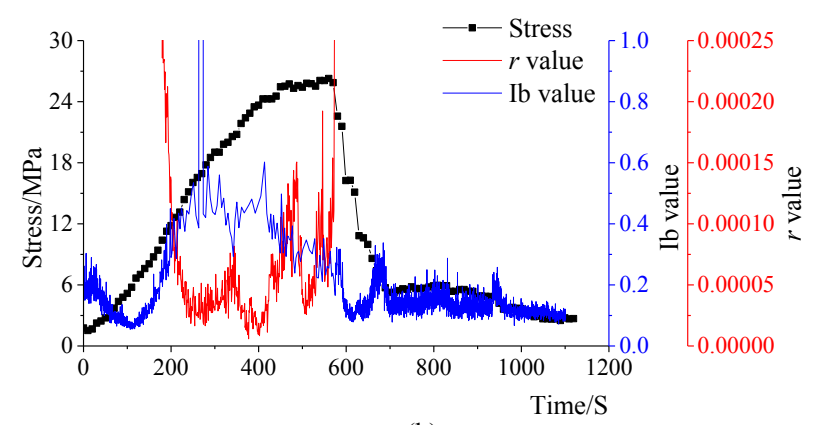

(b)

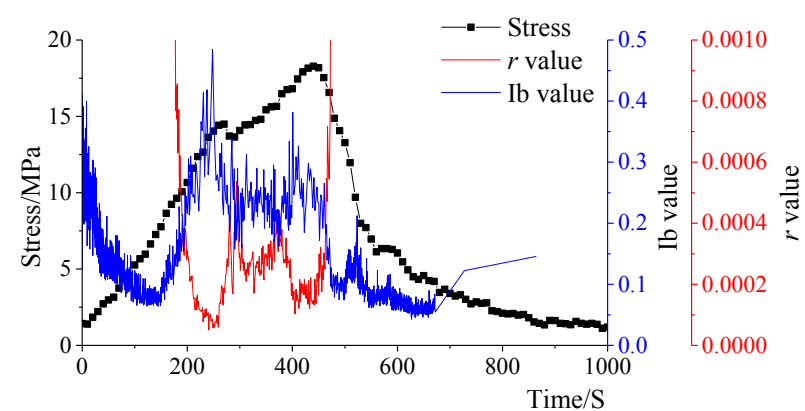

(c)

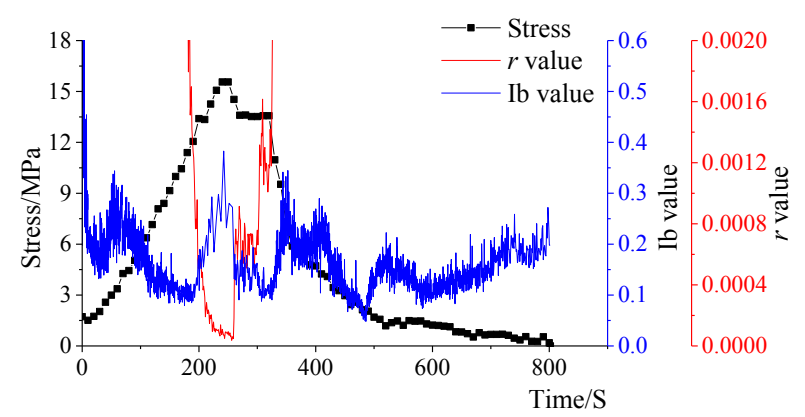

(d)

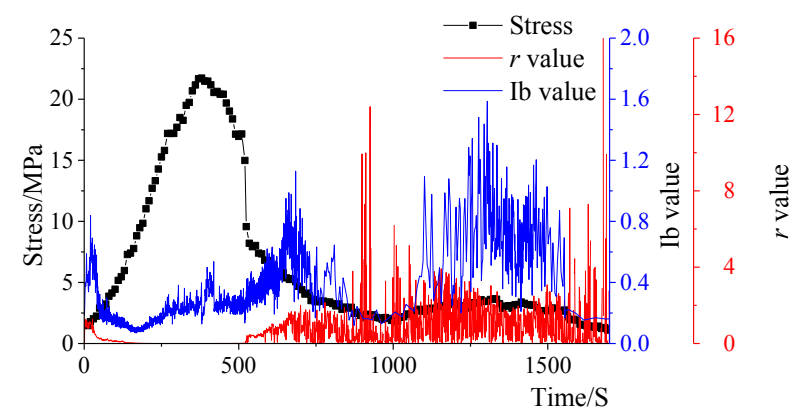

(e)

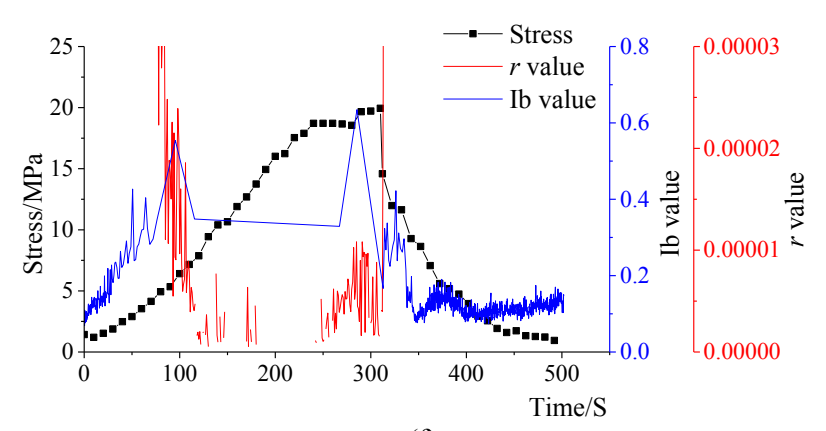

(f)

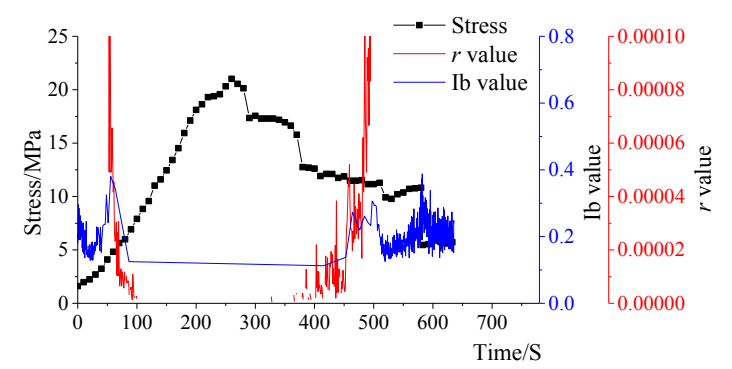

Fig. 8. Relationship between stress and AE parameters of samples in a coupling of the shear and extension modes. Self-Elaboration

(1) Extension mode: As shown in Figure 6, extension mode samples have obvious peak stress and their $r$ value curves demonstrate a typical U-shape distribution. In the pre-peak 
stress period, with increasing stress the $r$ value continuously decreases to the bottom of a U-shape, then increases dramatically after peak stress. For the $I b$ value, with increasing stress an alternate increase and decrease is displayed. With the relative quiescence period, the $I b$ value decreases significantly before the peak stress. If there is no relative quiescence period, the $I b$ value shows no significant decrease.

(2) Shear mode: As shown in Figure 7, most of the specimens have more than one shear fracture plane. Especially in the post-peak stress period, the interaction and shear friction of fractures makes the fracture mechanisms extremely complex. Furthermore, the relation between stress and time is also extremely complex. There is more than one peak stress during the whole shear failure process. For the first peak stress, the $r$ value curve has a significant U-shape similar to extension failure. After the first peak stress, the $r$ value increases dramatically, and the $I b$ value increases and decreases alternately and generally decreases near to the first peak stress.

(3) Coupling of the shear and extension modes: As shown in Figure 8, the relation between stress and time is also complex. Although there are numerous peak stresses, the largest peak stress is obvious. The $r$ value shows a significant $U$-shape in general. After every peak stress the $r$ value increases dramatically, before the first peak stress the $I b$ value decreases generally, and after the first peak stress the $I b$ value fluctuates with time.

\subsection{Analysis of $\mathrm{AE}$ characteristics in the whole process}

(1) The experimental results have shown that the minimum $r$ value is at the bottom of the U-shape before the peak stress and increases dramatically after the peak stress. This indicates that a small amount of AE events with high energy occur with microscopic fracture development before peak stress, and a large number of AE events with low energy occur with macroscopic fractures after peak stress.

The Ib value usually decreases before peak stress and there is no distinct rule for the post-peak stress period. Obviously the $r$ value can be used as a better indicator of rock failure than the $\mathrm{Ib}$ value. This point of view is consistent well with the results by Stephen et al. (2006).

(2) The experiments also show that there are some specimens with a relative quiescence period of $\mathrm{AE}$ under uniaxial compression regardless of failure mode. The $\mathrm{Ib}$ value fluctuates and only demonstrates a decrease generally near to the peak stress, and the $r$ value increases dramatically after peak stress.

(3) The Experiment's results indicate that the $r$ value decreases with the stress increase and holds a low value at the bottom of the U-shape before the peak stress. After the peak stress the $r$ value increases dramatically during the post-peak period. This could be used as an indicator of peak stress identification. In terms of the $\mathrm{Ib}$ value, this exhibits general decreasing trends before the peak stress, which can also be used as an assistant base for peak stress identification.

It is noteworthy that the $r$ value decreases with very little fluctuation and then increases sharply just after the first peak stress. If the $r$ value decreases and increases once more, the second time the $r$ value increases is just after the second peak stress, and so on.

\section{Conclusions}

Based on the above results the following major conclusions can be drawn.

(1) The phyllite rock samples under uniaxial compressive stress, due to localized stress concentrations around schistosity discontinuities, mainly fail in the extension mode with more fractures along a plane which almost parallel to the direction of compression. Other failures occur in the shear mode and a coupling of the shear and extension modes.

(2) The quiescence period of AE may appear before the peak stress for any failure mode. In addition, the $r$ value or the $\mathrm{Ib}$ value show no dramatic linear increment or decrement variation before the peak stress. It is difficult to determine the stress state before the peak stress depending on the $\mathrm{AE}$ monitoring data of the pre-peak stress period alone. Analysis of the $r$ value and the Ib value during the whole compression period makes it possible to determine whether the rock sample is in a pre- or in post-peak stress period.

(3) The plots of the Ib value show that the value variation with time is very complicated and usually decreases before the peak stress. The plots of the $r$ value can provide improved insight into the stress state. Before the peak stress the $r$ value decreases continuously and then keeps a relatively slow variation. After the peak stress the $r$ value increases dramatically. Hence, the $r$ value appears to be in a "U" distribution with time in a uniaxial compression process. The Ib value usually decreases while the $r$ value keeps a relatively low variation in the pre-peak stress period and the $r$ value increases sharply after the peak stress. This can be used as a basis to determine that the specimen is in a pre- or post-stress period. The achievements provide a scientific basis for in situ predictions of phyllite pillar failure through AE monitoring.

\section{Acknowledgements}

This work was supported by the National Natural Science Foundation of China under the project No. 51364012 and the China National Education Commission (413045) is gratefully acknowledged.

\section{References}

1. Shiotani, T., Evaluation of long-term stability for rock slope by means of acoustic emission technique. NDT \& E International, 39 (3), 2006, pp. 217-228.

2. Carpinteri, A., Lacidogna, G., and Niccolini, G., "Critical defect size distributions in concrete structures detected by the acoustic emission technique", Meccanica, 42 (8), 2008, pp. 349-363.

3. Li, X. B., and Liu, Z.X., "Research on chaos and intelligent identification of acoustic emission in rock mass", Chinese Journal of Rock Mechanics and Engineering, 24 (8), 2005, pp. 1296-1300.
4. Li, S.L., and Wang, Y.J., "Studies on acoustic emission characteristics of uniaxial compressive rock failure", Chinese Journal of Rock Mechanics and Engineering, 23 (15), 2004, pp. 2499-2503.

5. Yin, X.G., Li, S.L., and Tang, H.Y., "Study on strength fractal features of acoustic emission in process of rock failure", Chinese Journal of Rock Mechanics and Engineering, 24 (19), 2005, pp. 3512-3516.

6. Li, Y.H., Liu, J.P., and Zhao, X. D., "Study on b-value and fractal dimension of acoustic emission during rock failure process" Rock and Soil Mechanics, 30 (9), 2009, pp. 2559-2563. 
7. Lu, P.L., Wu, K.T., and Jiao, Y.B., "The experimental study of acoustic emission during creep of rocks", Acta seismologica sinaca, 13 (1), 1991, pp. 104-112.

8. Lockner, D., "The role of acoustic emission in the study of rock fracture", Int. J. Rock Mech. Min. Sci. \& Geomech, Abstr, 30 (7), 1993, pp. 883-899.

9. Stephen, A.H., Fiorenza, D.S., and Gioacchino, V., "Monitoring fracture propagation in a soft rock (Neapolitan Tuff) using acoustic emissions and digital images", Pure appl. Geophys, 163, 2006, pp. 2171-2204.

10. Přikryl, R. Lokajíček, T. Li, C, “Acoustic emission characteristics and failure of uniaxially stressed granitic rocks: the effect of rock fabric", Rock Mechanics and Rock Engineering, 36 (4), 2003, pp. 255-270.

11. Zhang, R., Xie, H.P., and Liu, J.F., "Experimental study on acoustic emission characteristics of rock failure under uniaxial multilevel loadings". Chinese Journal of Rock Mechanics and Engineering, 25 (12), 2006, pp. 2584-2588.

12. Xu, S.C., Feng, X.T., and Chen, B.R., "Experimental study of skarn under uniaxial cyclic loading and unloading test and acoustic emission characteristic", Rock and Soil Mechanics, 30 (10), 2009, pp. 2390-2394.

13. Jiang ,Y., Ge, X., and Ren, J. X., "Deformation rules and acoustic emission characteristics of rocks in process of fatigue failure", Chinese Journal of Rock Mechanics and Engineering, 23 (11) 2004, pp. 1810-1814.

14. S Zhao, K., He, W., and Gong, C., "Study on acoustic emission test of filling material failure process under cyclic loading and unloading condition", Transactions of Beijing institute of technology, 31(Supp.1), 2011, pp. 165-168.
15. Zhao, K., Yan, D.Q., and Zhong, C.H., "Comprehensive analysis method and experimental vertification for in-situ stress measurement by acoustic emission tests", Chinese Journal of geotechnical engineering, 34 (8), 2012, pp. 1403-1411.

16. Ji, H. G., Wang H. W., and Cao, S. Z., "Experimental research on frequency characteristics of acoustic emission signals under uniaxial compression of granite", Chinese Journal of Rock Mechanics and Engineering, 31(Supp.1), 2012, pp. 2900-2905.

17. Zeng, Z.W., Ma, J., and Liu, L.Q., "AE b-Value dynamic features during rockmass fracturing and their significances", Seismology and geology, 17 (1), 1995, pp. 7-11.

18. Santosh, G., Shah, J.M., and Chandra, K., "Use of acoustic emissions in flexural fatigue crack growth studies on concrete", Engineering Fracture Mechanics, 87 (6), 2012, pp. 36-47.

19. Lavrov, A. V., and Shkuratnik V. L., "Deformation and fracture induced acoustic emission in Rocks", Acoustical Physics 51 (Supp.1), 2005, pp. 2-11.

20. Shiotani, T., Fujii, K., Aoki, T., and Amou, K., "Evaluation of progressive failure using $\mathrm{AE}$ sources and improved b-value on slope model tests", Prog Acoust Emiss VII, 7, 1994, pp. 529-534.

21. Shiotani, T., and Ohtsu, M., "Prediction of slope failure based on AE activity. Vahaviolos, S.J. (Ed.)" ASTM special technical publication: Philadelphia, press, 1999, pp. 156-172.

22. Yo-Seph, B., Myung, S., and Suk-Chun, K., "A study on using acoustic emission in rock slope with difficult ground focused on rainfall”, Geosciences Journal, 16 (4), 2012, pp. 435-445.

23. Zheng, D., and Ju, N.P., "Scanning electronic microscope tests for rock micro-rupture mechanism and fracture characteristic of phyllite", Journal of engineering geology, 19 (3), 2011, pp. 317322.

24. Szwedzicki, T., "A hypothesis on modes of failure of rock samples tested in uniaxial compression", Rock Mechanics and Rock Engineering, 40 (1), 2007, pp. 97-104. 